\title{
Redaksjonsråd - Editorial board
}

Vilhjalmur Arnason, HI, Reykjavik

Alexander Cappelen, NHH, Bergen

Gøran Collste, LiU, Linköping

Linn Getz, NTNU, Trondheim

Siri Gloppen, CMI \& UiB, Bergen

Lars Grue, NOVA, Oslo

Mette Hartlev, KU, København

Bjørn Hofmann, UiO, Oslo

Cathrine Holst, UiB, Bergen

Helge Høibraaten, NTNU, Trondheim

Matthias Kaiser, UiB, Bergen

Bjørn K. Myskja, NTNU, Trondheim

Torben Hviid Nielsen, UiO, Oslo

Rune Nydal, NTNU, Trondheim

Ulla Schmidt, KIFO \& UiO, Oslo

John-Arne Skolbekken, NTNU, Trondheim

Jan Helge Solbakk, UiO, Oslo

Berge Solberg, NTNU, Trondheim

Roger Strand, UiB, Bergen

Knut Sørensen, NTNU, Trondheim

Arne Johan Vetlesen, UiO, Oslo

Jon Wetlesen, UiO, Oslo

Truls Wyller, NTNU, Trondheim

Audun Øfsti, NTNU, Trondheim

Knut Ågotnes, UiB, Bergen

Simo Vehmas, University of Helsinki

Homepage: http://www.ntnu.no/ojs/index.php/etikk_i_praksis/index

Publisher: Programme for Applied Ethics, Department of Philosophy \& Religious Studies Norwegian University of Science and Technology

NTNU, 7491 Trondheim, Norway

redaksjon.eip@gmail.com

Tel: +4773596535

Fax: +4773596460

ISSN (online): 1890-4009

Print ISSN: 1890-3991

Languages: Norwegian, Swedish, Danish and English

Indexed in DOAJ, Thomson Reuters (ISI), EbscoHost, Philosopher's Index and Scopus 